Article

\title{
Inverse Problems for Degenerate Fractional Integro-Differential Equations
}

\author{
Mohammed Al Horani ${ }^{1}$, Mauro Fabrizio ${ }^{2}{ }^{\mathbb{D}}$, Angelo Favini ${ }^{2, *}$ and Hiroki Tanabe ${ }^{3}$ \\ 1 Department of Mathematics, The University of Jordan, Amman 11942, Jordan; horani@ju.edu.jo \\ 2 Dipartimento di Matematica, Università di Bologna, Piazza di Porta S. Donato 5, 40126 Bologna, Italy; \\ mauro.fabrizio@unibo.it \\ 3 Takarazuka, Hirai Sanso 12-13, Osaka 665-0817, Japan; bacbx403@jttk.zaq.ne.jp \\ * Correspondence: angelo.favini@dm.unibo.it
}

Received: 19 February 2020; Accepted: 23 March 2020; Published: 3 April 2020

\begin{abstract}
This paper deals with inverse problems related to degenerate fractional integro-differential equations in Banach spaces. We study existence, uniqueness and regularity of solutions to the problem, claiming to extend well known studies for the case of non-fractional equations. Our method is based on transforming the inverse problem to a direct problem and identifying the conditions under which this direct problem has a unique solution. The conditions under which the unique strict solution can be compared with the case of a mild solution, obtained in previous studies under quite restrictive requirements, are on the underlying functions. Applications from partial differential equations are given to illustrate our abstract results.
\end{abstract}

Keywords: fractional derivative; abstract Cauchy problem; evolution equation; inverse problem

MSC: 34K29; 34K30; 34K37; 35R11; 35R30

\section{Introduction}

This paper is devoted to inverse problems for degenerate integro-differential equations. The basic aim is to introduce the study of inverse problems related to degenerate fractional integro-differential equations, extending the previous results of Al Horani and Favini [1], Al Horani et al. [2-5] and Favaron et al. [6]. Completely different methods were used by Fedorov and Ivanova [7], Sviridyuk and Fedorov [8] together with many papers from their school, see References [7-13], see also [14-21] and the monograph of Bazhlekova [22]. Let us also remind, in particular [23,24] where the authors considered equations of Sobolev type, with nonlocal conditions, of the form

$$
\begin{aligned}
& D^{q}(B u(t))=A u(t)+f\left(t, u(t), \int_{0}^{t} k(t, s, u(s)) d s\right), t \in J=[0, \tau], \\
& u(0)=u_{0}
\end{aligned}
$$

with Riemann-Liouville fractional derivative $D^{q}, 0<q<1, A, B$ being closed linear operators from $X$ into $Y, X, Y$ are two Banach spaces, $D(B) \subseteq D(A), B$ is bijective so that $B^{-1}: Y \rightarrow D(B)$ is continuous, $f: J \times X^{2} \rightarrow X_{B^{-1} A} \equiv D\left(B^{-1} A\right), k: \Omega \times X \rightarrow X$ are continuous, being $\Omega=\{(t, s): 0 \leq s \leq t \leq \tau\}$. If we use, for sake of brevity,

$$
K u(t)=\int_{0}^{t} k(t, s, u(s)) d s,
$$


they define a mild solution $u$ of (1)-(2) as a function $u \in C(J ; X)$ such that $\int_{0}^{t} u(s)(t-s)^{q-1} \in$ $D\left(B^{-1} A\right)$ for all $t \in J$, and

$$
u(t)=u_{0}+\frac{1}{\Gamma(q)} \int_{0}^{t} \frac{B^{-1} A u(s)}{(t-s)^{q-1}} d s+\frac{1}{\Gamma(q)} \int_{0}^{t} \frac{B^{-1} f(s, u(s), K u(s))}{(t-s)^{q-1}} d s .
$$

In order to obtain an existence result, the authors of Reference [23] were compelled to require that ( see Reference [23], Theorem 3.2, p. 3409) $u_{0} \in D\left(B^{-1} A\right), f: J \times X^{2} \rightarrow X_{B^{-1} A}$ is completely continuous and there exists a positive constant $L_{1}$ such that

$$
\left\|f\left(t, x_{1}, y_{1}\right)-f\left(t, x_{2}, y_{2}\right)\right\|_{D\left(B^{-1} A\right)} \leq L_{1}\left(\left\|x_{1}-x_{2}\right\|_{X}+\left\|y_{1}-y_{2}\right\|_{X}\right),
$$

$k: \Omega \times X \rightarrow D\left(B^{-1} A\right)$ is continuous and there is a constant $L_{2}>0$ such that

$$
\left\|\int_{0}^{t}\left[k\left(t, s, x_{1}\right)-k\left(t, s, x_{2}\right)\right] d s\right\|_{D\left(B^{-1} A\right)} \leq L_{2}\left\|x_{1}-x_{2}\right\|_{X}
$$

Moreover, it is assumed, in order to apply fixed point arguments, that a certain obtained constant is less than 1 . Then problem (1) and (2) admits a mild solution on J. This result shows how many restrictive assumptions must be done to obtain only a mild solution to a weakly degenerate equation (recall that it is assumed $B^{-1}: Y \rightarrow D(B)$ is continuous).

Our problem consists in studying existence, uniqueness and regularity of a pair $(y, f) \in$ $C([0, \tau] ; D(L)) \times C([0, \tau] ; \mathbb{C})$ solving, in a strict sense, the integro-differential problem

$$
\begin{aligned}
& D_{t}^{\tilde{\alpha}}(M y(t))=L y(t)+\int_{0}^{t} k(t-s) L_{1} y(s) d s+f(t) z+h(t), t \in[0, \tau], \\
& M y(0)=M y_{0}(=0 \text { for simplicity }) \\
& \Phi[M y(t)]=g(t), t \in[0, \tau]
\end{aligned}
$$

where $L, L_{1}, M$ are closed linear operators acting on the complex Banach space $X, 0<\tilde{\alpha}<1$, $k \in C([0, \tau] ; \mathbb{C}), D(L) \subseteq D\left(L_{1}\right) \cap D(M), z \in X, h \in C([0, \tau] ; X), \Phi \in X^{*}$, the dual space of $X$, $f \in C([0, \tau] ; \mathbb{C}), \Phi\left[M y_{0}\right]=g(0)$ being the necessary compatibility relation to be satisfied in advance. Analogous problems with $\tilde{\alpha}=1$ have been considered by many authors, above all for $M=I$, the identity operator, see in particular $[15,25]$. The case for $\tilde{\alpha}=1$ without the integral sign has been considered recently in Reference [6], see also Al Horani et al. [3-5]. Also one can find some related results in Reference [7] where the authors extended, on the grounds of Reference [8] and the previous results of Favini and Lorenzi [26], see also Favini and Yagi [27], pp. 157-162.

The plan of this paper is as follows. In Section 2 we recall previous results on possibly degenerate differential and integro-differential equations. Section 3 is devoted to the preliminaries for the general case $\tilde{\alpha} \in(0,1)$. In Section 4 we consider the special case $\tilde{\alpha}=1$. Section 5 is related to the main case $\tilde{\alpha} \in(0,1)$. Section 6 contains some examples and applications.

It must be noted that the conditions on $f$ and $k$ in Reference [23] are very restrictive and one expects that such conditions can imply strict solutions. At this purpose, we recall that our required strict solution $y(t)$ is defined on the whole interval $[0, \tau]$ and $L y, D_{t}^{\tilde{\alpha}} M y$ have convenient Holder regularity in time.

More general problems like

$$
\begin{aligned}
& D_{t}^{\tilde{\alpha}}(M y(t))=L y(t)+\sum_{i_{1}=1}^{n_{1}} \int_{0}^{t} k_{i_{1}}(t-s) L_{i_{1}} y(s) d s+\sum_{i_{2}=1}^{n_{2}} f_{i_{2}}(t) z_{i_{2}}+h(t), t \in[0, \tau], \\
& M y(0)=0\left(\text { or } M y(0)=M y_{0}\right) \\
& \Phi_{i_{2}}[M y(t)]=g_{i_{2}}(t), \quad t \in[0, \tau], i_{2}=1,2, \ldots, n_{2}
\end{aligned}
$$


could be of interest in the future.

\section{Previous Results and Preliminaries}

This section is devoted to recall previous results that shall be used in the sequel. We begin with the following lemma from [15].

Lemma 1. Consider the problem

$$
\begin{aligned}
& \frac{d}{d t}(M y(t))=L y(t)+\int_{0}^{t} k(t-s) L_{1} y(s) d s+f(t), t \in[0, \tau], \\
& M y(0)=M y_{0},
\end{aligned}
$$

where $y_{0} \in D(L) \subseteq D(M) \cap D\left(L_{1}\right), 0 \in \rho(L), z M-L$ has a bounded inverse for any $z$ in the region

$$
\Sigma_{\alpha}=\left\{z \in \mathbb{C}: \operatorname{Re} z \geq-C(1+|\operatorname{Im} z|)^{\alpha}\right\}
$$

with

$$
\left\|M(z M-L)^{-1}\right\|_{\mathcal{L}(X)} \leq C(1+|\lambda|)^{-\beta}, \lambda \in \Sigma_{\alpha}, 0<\beta \leq \alpha \leq 1, \alpha+\beta>1,
$$

$f \in C^{\theta}([0, \tau] ; X), k \in C^{\theta}([0, \tau] ; \mathbb{C}), f(0)+L y_{0} \in R(T)=R\left(M L^{-1}\right), 2-\alpha-\beta<\theta<1$. Then problem (6)-(7) admits a unique global strict solution $y \in C^{\omega}([0, \tau] ; D(L)), M y \in C^{1+\omega}([0, \tau] ; X)$, $\omega=\alpha+\beta+\theta-2$.

The following result is important, see Reference [6].

Lemma 2. Let $A=L M^{-1}, A_{1}=\left(L+L_{1}\right) M^{-1}$ be two multivalued linear operators in $X$, where $L_{1} \in$ $\mathcal{L}\left(D(L), X_{A}^{\bar{\theta}}\right)$, with $\bar{\theta}>1-\beta, M, L, L_{1}$ being closed linear operators on $X$, and for all $\varphi \in(0,1)$, $X_{A}^{\varphi}=X_{A}^{\varphi, \infty}$ denotes the Banach space

$$
X_{A}^{\varphi}=\left\{u \in X, \sup _{t>0} t^{\varphi}\left\|A^{0}(t-A)^{-1} u\right\|_{X}=\|u\|_{X_{A}^{\varphi}}<\infty\right\},
$$

with $A^{\circ}(t-A)^{-1}:=-I+t(t-A)^{-1}$. Then for all $\theta \in(0,1)$

$$
X_{A}^{\theta}=X_{A_{1}}^{\theta}=X_{\left(k M+L+L_{1}\right) M^{-1}}^{\theta}, k \text { large. }
$$

Lemma 3. Let $\alpha+\beta>1,2-\alpha-\beta<\theta<1, D(L) \subseteq D(M) \cap D\left(L_{1}\right)$ where $M, L, L_{1}$ are closed linear operators on $X, A=L M^{-1}, T=A^{-1}=M L^{-1}$. If $y_{0} \in D(L), h \in C^{\theta}([0, \tau] ; X), g \in C^{1+\theta}([0, \tau] ; \mathbb{C})$, $k \in C^{\theta}([0, \tau] ; \mathbb{C}), h(0)+L y_{0} \in R(T)=D(A), \Phi[z] \neq 0, \Phi \in X^{*}$, then the inverse problem

$$
\begin{aligned}
& \frac{d}{d t}(M y(t))=L y(t)+\int_{0}^{t} k(t-s) L_{1} y(s) d s+f(t) z+h(t), t \in[0, \tau] \\
& M y(0)=M y_{0} \\
& \Phi[M y(t)]=g(t), t \in[0, \tau]
\end{aligned}
$$

admits a unique strict solution, that is,

$$
(y, f) \in C^{\theta-2+\alpha+\beta}([0, \tau] ; D(L)) \times C^{\theta-2+\alpha+\beta}([0, \tau] ; \mathbb{C}), \quad M y \in C^{\theta-1+\alpha+\beta}([0, \tau] ; X) .
$$


Notice that when we apply $\Phi$ to both sides of Equation (8) we get

$$
g^{\prime}(t)=\Phi[L y(t)]+\int_{0}^{t} k(t-s) \Phi\left[L_{1} y(s)\right] d s+f(t) \Phi[z]+\Phi[h(t)]
$$

so that necessarily

$$
f(t)=\frac{1}{\Phi[z]}\left\{g^{\prime}(t)-\Phi[L y(t)]-\int_{0}^{t} k(t-s) \Phi\left[L_{1} y(s)\right] d s-\Phi[h(t)]\right\} .
$$

Therefore, (8) takes the form

$$
\begin{aligned}
\frac{d}{d t}(M y(t))= & L y(t)+L_{2} y(t)+\int_{0}^{t} k(t-s) L_{1} y(s) d s-\frac{1}{\Phi[z]} \int_{0}^{t} k(t-s) \Phi\left[L_{1} y(s)\right] z d s \\
& +\frac{g^{\prime}(t)}{\Phi[z]} z-\frac{\Phi[h(t)]}{\Phi[z]} z+h(t)
\end{aligned}
$$

where $L_{2}$ is defined by

$$
D\left(L_{2}\right)=D(L),\left(L_{2} y\right)(t)=-\frac{\Phi[L y(t)]}{\Phi[z]} z .
$$

Notice that $L y(t)-\frac{\Phi[L y(t)]}{\Phi[z]} z=\left(L+L_{2}\right) y$ in the inverse problem leads to assume that $h(0)+L y_{0}-\frac{\Phi\left[L y_{0}(t)\right]}{\Phi[z]} z \in R(T)$, a.e., $f(0)+L y_{0} \in R(T), z \in R(T)$. These conditions are strongly restrictive. More precise and better results canceling, in particular, $f(0)+L y_{0}$ have been obtained by Favaron-Favini, see Reference [25], Theorem 48.

Lemma 4. Assume that L has a bounded inverse, $y_{0} \in D(L), 5 \alpha+2 \beta>6$,

operators $L, M$ satisfy

$$
\left\|M(\lambda M-L)^{-1}\right\|_{\mathcal{L}(X)} \leq \frac{c}{(|\lambda|+1)^{\beta}}
$$

for any $\lambda \in \Sigma_{\alpha}:=\left\{z \in \mathbb{C}: \operatorname{Re} z \geq-c(1+|\operatorname{Im} z|)^{\alpha}, \quad c>0,0<\beta \leq \alpha \leq 1\right\} . \quad\left(\lambda_{0} M+L\right) y_{0}+$ $f(0) \in X_{A}^{\varphi}, A=L M^{-1},\left(z_{1}, \ldots, z_{n_{2}}\right) \in \prod_{i_{2}=1}^{n_{2}} X_{A}^{\gamma_{i_{2}}}, k_{i_{1}} \in C^{\eta_{i_{1}}}([0, \tau] ; \mathbb{C}), h_{i_{2}} \in C^{\sigma_{i_{2}}}([0, \tau] ; \mathbb{C}), \gamma_{i_{2}}, \varphi \in$ $(5-3 \alpha-2 \beta, 1), \eta_{i_{1}}, \sigma_{i_{2}} \in((3-2 \alpha-\beta) / \alpha, 1), i_{l}=1, \ldots, n_{l}, l=1,2$. Let $\gamma=\min _{i_{2}=1, \ldots, n_{2}}\left\{\gamma_{i_{2}}, \varphi\right\}$, $\bar{\tau}=\min _{i_{l}=1, \ldots, n_{l}, l=1,2}\left\{\eta_{i_{1}}, \sigma_{i_{2}},(\alpha+\beta+\gamma-2) / \alpha\right\}$. Then for every fixed $\delta \in I_{\alpha, \beta, \bar{\tau}}$ where

$$
\begin{aligned}
& I_{\alpha, \beta, \bar{\tau}}= \begin{cases}\left(\frac{3-2 \alpha-\beta}{\alpha}, \bar{\tau}\right] & \text { if } \bar{\tau} \in\left(\frac{3-2 \alpha-\beta}{\alpha}, \frac{1}{2}\right) \\
\left(\frac{3-2 \alpha-\beta}{\alpha}, \frac{1}{2}\right) & \text { if } \bar{\tau} \in\left[\frac{1}{2}, 1\right)\end{cases} \\
& \frac{d}{d t}(M y(t))=\left[\lambda_{0} M+L\right] y(t)+\sum_{i_{1}=1}^{n_{1}} \int_{0}^{t} k_{i_{1}}(t-s) L_{i_{1}} y(s) d s+\sum_{i_{2}=1}^{n_{2}} h_{i_{2}}(t) z_{i_{2}}+f(t), t \in[0, \tau], \\
& M y(0)=M y_{0}
\end{aligned}
$$

admits a unique strict solution $y \in C^{\delta}([0, \tau] ; D(L))$ such that $L y, D_{t} M y \in C^{\delta}([0, \tau] ; X)$, provided that $f \in C^{\mu}([0, \tau] ; X), \mu \in[\delta+(3-2 \alpha-\beta) / \alpha, 1)$. Here $\lambda_{0}$ is a fixed constant such that $\lambda_{0} M+L$ has a bounded inverse. 
In particular, the simplest case

$$
\begin{aligned}
& \frac{d}{d t}(M y(t))=\left(\lambda_{0} M+L\right) y(t)+\int_{0}^{t} k(t-s) L_{1} y(s) d s+f(t) z+h(t), \quad t \in[0, \tau], \\
& (M y)(0)=M y_{0}
\end{aligned}
$$

admits a unique strict solution $y$, for any $y_{0} \in D(L), 5 \alpha+2 \beta>6,\left\|M(\lambda M-L)^{-1}\right\|_{\mathcal{L}(X)} \leq \frac{c}{(|\lambda|+1)^{\beta}}$ for any $\lambda \in \Sigma_{\alpha}:=\left\{z \in \mathbb{C}: \operatorname{Re} z \geq-c(1+|\operatorname{Im} z|)^{\alpha}\right\}, c>0,0<\beta \leq \alpha \leq 1,\left(\lambda_{0} M+L\right) y_{0}+h(0) \in$ $X_{A}^{\varphi}, z \in X_{A}^{\gamma}, k \in C^{\eta}([0, \tau] ; \mathbb{C}), f \in C^{\sigma}([0, \tau] ; \mathbb{C}), \eta, \sigma \in((3-2 \alpha-\beta) / \alpha, 1), \gamma, \varphi \in(5-3 \alpha-2 \beta, 1)$, $\bar{\gamma}=\min \{\gamma, \varphi\}$. Such a solution $y \in C^{\delta}([0, \tau] ; D(L))$ and $L y, D_{t} M y \in C^{\delta}([0, \tau] ; X)$ provided that $h \in C^{\mu}([0, \tau] ; X), \mu \in\left[\delta+\frac{3-2 \alpha-\beta}{\alpha}, 1\right), \bar{\tau}=\min \{\eta, \sigma,(\alpha+\beta+\bar{\gamma}-2) / \alpha\}$ for any $\delta \in I_{\alpha, \beta, \bar{\tau}}$ where

$$
I_{\alpha, \beta, \bar{\tau}}= \begin{cases}\left(\frac{3-2 \alpha-\beta}{\alpha}, \bar{\tau}\right] & \text { if } \bar{\tau} \in\left(\frac{3-2 \alpha-\beta}{\alpha}, \frac{1}{2}\right) \\ \left(\frac{3-2 \alpha-\beta}{\alpha}, \frac{1}{2}\right) & \text { if } \bar{\tau} \in\left[\frac{1}{2}, 1\right) .\end{cases}
$$

The following result of Favaron-Favini-Tanabe [6] holds

Lemma 5. Let $M, L, D(L) \subseteq D(M)$ be closed single-valued linear operators in $X$ such that $0 \in \rho(L)$ and let $\Psi_{i} \in X^{*}, i=1, \ldots, N, N \in \mathbb{N}$. Assume also

$\left(\mathcal{H}_{1}\right)\left\|M(\lambda M-L)^{-1}\right\|_{\mathcal{L}(X)} \leq \frac{c}{(|\lambda|+1)^{\beta}}$ for any $\lambda \in \Sigma_{\alpha}:=\left\{\xi \in \mathbb{C}: \operatorname{Re} \xi \geq-c(1+|\operatorname{Im} \xi|)^{\alpha}, c>\right.$ $0,0<\beta \leq \alpha \leq 1\}$.

$\left(\mathcal{H}_{2}\right) v_{0} \in D(L), y_{0}=L v_{0}+h(0) \in X_{A}^{\gamma_{0}, \infty}=X_{A}^{\gamma_{0}}, \quad A=L M^{-1},\left(y_{0}, y_{1}, \ldots, y_{N}\right) \in \prod_{i=0}^{N} X_{A}^{\gamma_{i}}, \gamma_{i} \in$ $(5-3 \alpha-2 \beta, 1), i=1,2, \ldots, N$.

$\left(\mathcal{H}_{3}\right) h \in C^{\mu_{0}}([0, \tau] ; X), \mu_{0}-1 / 2 \in[(3-2 \alpha-\beta) / \alpha, 1)$

$\left(\mathcal{H}_{4}\right) g_{i} \in C^{1+\mu}([0, \tau] ; \mathbb{C}), \mu \in((3-2 \alpha-\beta) / \alpha, 1), i=1,2, \ldots, N$,

$$
U=\left[\begin{array}{lll}
\Psi_{1}\left[y_{1}\right] & \ldots & \Psi_{1}\left[y_{N}\right] \\
\Psi_{N}\left[y_{1}\right] & \ldots & \Psi_{N}\left[y_{N}\right]
\end{array}\right]
$$

is an invertible matrix. Let $\gamma=\min _{i=1, \ldots, N} \gamma_{i}, \bar{\tau}=\min (\mu,(\alpha+\beta+\gamma-2) / \alpha)$, where $\alpha, \beta$ as in $\left(\mathcal{H}_{1}\right)$. Then the degenerate identification problem

$$
\begin{aligned}
& D_{t}(M v(t))=L v(t)+\sum_{i=1}^{N} f_{i}(t) y_{i}+h(t), t \in[0, \tau], \\
& M v(0)=M v_{0}, \\
& \Psi_{i}[M v(t)]=g_{i}(t), \quad t \in[0, \tau], i=1,2, \ldots, N, \\
& \Psi_{i}\left[M v_{0}\right]=g_{i}(0), i=1,2, \ldots, N
\end{aligned}
$$

for each fixed $\delta \in I_{\alpha, \beta, \bar{\tau}}$, admits a unique strict global solution $\left(v, f_{1}, \ldots, f_{N}\right)$ such that $v \in C^{\delta}([0, \tau] ; D(L))$, $M v \in C^{1+\delta}([0, \tau] ; X), f_{i} \in C^{\delta}([0, \tau] ; \mathbb{C}), i=1, \ldots, N$. 


\section{Introduction to the Case $\tilde{\alpha} \in(0,1)$}

In order to handle the case $\tilde{\alpha} \in(0,1)$, we recall that

$$
\begin{aligned}
& D_{t}^{\tilde{\alpha}}(M y(t))=L y(t)+f(t), t \in[0, \tau], \\
& (M y)(0)=0,
\end{aligned}
$$

was recently considered by $\mathrm{Al}$ Horani et al., see Reference [3], where the authors took into account the abstract results of Favini and Yagi [27] generalized by Favini et al. [15].

Assume $\left(\mathcal{H}_{1}\right)$ to hold together with the hypothesis that the closed linear operator $B$ has a resolvent $(z-B)^{-1}$ for all $z \in \mathbb{C}: \operatorname{Re} z<a, a>0$ such that $\left\|(\lambda-B)^{-1}\right\|_{\mathcal{L}(E)} \leq C(|\operatorname{Re} \lambda|+1)^{-1}, \operatorname{Re} \lambda<a, E$ is a complex Banach space, $A=L M^{-1}, D(A)=M(D(L))$ and $B$ commute in the sense $B^{-1} A^{-1}=A^{-1} B^{-1}$

Proposition 1. Suppose that $\alpha+\beta>1,2-\alpha-\beta<\theta<1$. Then under the hypotheses above, equation $B M u=L u+f$ admits a unique strict solution $u$ such that $L u, B M u \in(E, D(B))_{\omega, p}, \omega=\theta-2+\alpha+\beta$, provided that $f \in(E, D(B))_{\theta, p}, 1 \leq p \leq \infty$.

If $X$ is a complex Banach space, introduce operator $B_{X}$ by

$$
\begin{aligned}
& B_{X}:\left\{v \in C^{1}([0, \tau] ; X) ; v(0)=0\right\} \longrightarrow C([0, \tau] ; X) \\
& B_{X} v=D_{t} v
\end{aligned}
$$

It is well known that $\rho\left(B_{X}\right)=\mathbb{C}$ and $B_{X}$ is a positive operator in $C([0, \tau] ; X)$ of type $\pi / 2$. Powers for $B_{X}$ are defined as follows

$$
B_{X}^{-\delta} f(t)=\frac{1}{\Gamma(\delta)} \int_{0}^{t}(t-s)^{\delta-1} f(s) d s
$$

for all $\delta>0, f \in C([0, \tau] ; X)$ and any $t \in(0, \tau]$. Since $B_{X}^{-\delta}$ is injective, one defines for $\delta>0$

$$
B_{X}^{\delta}=\left(B_{X}^{-\delta}\right)^{-1} .
$$

It is known that if $\delta \in(0,2), B_{X}^{\delta}$ is positive of type $\frac{\delta \pi}{2}$. Moreover, the following interpolation result holds.

Proposition 2. Let $0 \leq \alpha_{0}<\alpha_{1}, \xi \in(0,1),(1-\xi) \alpha_{0}+\xi \alpha_{1} \notin \mathbb{N}$. Then

$$
\begin{gathered}
\left(D\left(B_{X}^{\alpha_{0}}\right), D\left(B_{X}^{\alpha_{1}}\right)\right)_{\xi, \infty}=\left\{f \in C^{(1-\xi) \alpha_{0}+\xi \alpha_{1}}([0, \tau] ; X), f^{(k)}(0)=0, \text { for all } k \in \mathbb{N}_{0},\right. \\
\left.k<(1-\xi) \alpha_{0}+\xi \alpha_{1}, \mathbb{N}_{0}=\mathbb{N} \cup\{0\}\right\}
\end{gathered}
$$

so that

$$
\left(C([0, \tau] ; X), D\left(B_{X}^{\alpha_{1}}\right)\right)_{\xi, \infty}=\left\{f \in C^{\xi \alpha_{1}}([0, \tau] ; X), f^{(k)}(0)=0, \text { for all } k \in \mathbb{N}_{0}, k<\xi \alpha_{1}\right\} .
$$

It follows that since operator $B_{X}^{\tilde{\alpha}}$ satisfies the spectral property described above, for any $\tilde{\alpha} \in(0,1]$

$$
\begin{aligned}
& D_{t}^{\tilde{\alpha}}(M u(t))-L u(t)=f(t), 0 \leq t \leq \tau \\
& (M u)(0)=0
\end{aligned}
$$


admits a strict solution $u$ such that $L u, \quad D_{t}^{\tilde{\alpha}} M u \in C^{\tilde{\alpha} \omega}([0, \tau] ; X), \quad(L u)(0)=0=D_{t}^{\tilde{\alpha}} M u(0)$, $\alpha+\beta>1,2-\alpha-\beta<\theta<1, \omega=\alpha+\beta+\theta-2$, provided that $f \in\left(C([0, \tau] ; X), D\left(B_{X}^{\tilde{\alpha}}\right)\right)_{\theta, \infty}=$ $\left\{f \in C^{\tilde{\alpha} \theta}([0, \tau] ; X), f(0)=0\right\}$.

\section{The Integro-Differential Problem for $\tilde{\alpha}=1$}

Of concern is the inverse problem

$$
\begin{aligned}
& D_{t}(M y(t))=\left(z_{0} M+L\right) y(t)+\int_{0}^{t} k(t-s) L_{1} y(s) d s+f(t) z+h(t), \quad t \in[0, \tau], \\
& M y(0)=M y_{0}, y_{0} \in D(L) \\
& \Phi[M y(t)]=g(t), \quad t \in[0, \tau], \Phi\left[M y_{0}\right]=g(0),
\end{aligned}
$$

$D(L) \subseteq D\left(L_{1}\right) \cap D(M), k \in C([0, \tau] ; \mathbb{C})$. The unknown is the pair $(y, f), f \in C([0, \tau] ; \mathbb{C})$. In order to avoid problems for the sum of closed operators, we assume that $z_{0} M+L$ has a bounded inverse and introduce the new variable $x=\left(z_{0} M+L\right) y$. Then (11)-(13) takes the form

$$
\begin{aligned}
& D_{t} M\left(z_{0} M+L\right)^{-1} x=x+\int_{0}^{t} k(t-s) L_{1}\left(z_{0} M+L\right)^{-1} x(s) d s+f(t) z+h(t), t \in[0, \tau], \\
& \left(M\left(z_{0} M+L\right)^{-1} x\right)(0)=M y_{0}=M\left(z_{0} M+L\right)^{-1} x_{0}, x_{0}=\left(z_{0} M+L\right) y_{0} \\
& \Phi\left[M\left(z_{0} M+L\right)^{-1} x(t)\right]=g(t), t \in[0, \tau] .
\end{aligned}
$$

One may note that all involved operators are bounded. Observe also

$$
\begin{aligned}
M\left(z_{0} M+L\right)^{-1}\left[\lambda M\left(z_{0} M+L\right)^{-1}-I\right]^{-1} & =M\left(z_{0} M+L\right)^{-1}\left(z_{0} M+L\right)\left[\lambda M-z_{0} M-L\right]^{-1} \\
& =M\left(\left(\lambda-z_{0}\right) M-L\right)^{-1}
\end{aligned}
$$

and that

$$
\left\|M\left(z_{0} M+L\right)^{-1}\left[\lambda M\left(z_{0} M+L\right)^{-1}-I\right]\right\|_{\mathcal{L}(X)}=\left\|M\left(\left(\lambda-z_{0}\right) M-L\right)^{-1}\right\|_{\mathcal{L}(X)} \leq C(1+|\lambda|)^{-\beta}
$$

for all $\lambda \in \Sigma_{\alpha}$. In this case $A=\left(z_{0} M+L\right) M^{-1}$, as expected. Applying $\Phi$ to both sides of Equation (14), we get

$$
g^{\prime}(t)=\Phi[x]+\int_{0}^{t} k(t-s) \Phi\left[L_{1}\left(z_{0} M+L\right)^{-1} x(s)\right] d s+f(t) \Phi[z]+\Phi[h(t)] .
$$

If $\Phi[z] \neq 0$, then

$$
f(t)=\frac{1}{\Phi[z]}\left\{g^{\prime}(t)-\Phi[x]-\int_{0}^{t} k(t-s) \Phi\left[L_{1}\left(z_{0} M+L\right)^{-1} x(s)\right] d s-\Phi[h(t)]\right\} .
$$

Therefor, we get a direct problem, precisely,

$$
\begin{aligned}
& D_{t} M\left(z_{0} M+L\right)^{-1} x= \\
& x-\frac{\Phi[x(t)]}{\Phi[z]} z+\int_{0}^{t} k(t-s)\left[L_{1}\left(z_{0} M+L\right)^{-1} x(s)-\frac{\Phi\left[L_{1}\left(z_{0} M+L\right)^{-1} x(s)\right]}{\Phi[z]} z\right] d s+ \\
& \quad \frac{g^{\prime}(t)}{\Phi[z]} z-\frac{\Phi[h(t)]}{\Phi[z]} z+h(t), \quad t \in[0, \tau] .
\end{aligned}
$$


One applies Lemma 4 and notice that $z M\left(z_{0} M+L\right)^{-1}-I-\frac{\Phi[\cdot]}{\Phi[z]} z$ has the same spectral properties of $z M\left(z_{0} M+L\right)^{-1}-I$, provided that $z \in X_{A}^{\bar{\theta}}$ for some $\bar{\theta}>1-\beta$, see Favini and Tanabe [16]. Thus our assumptions reduce to $5 \alpha+2 \beta>6, z \in X_{A^{\prime}}^{\gamma} k \in C^{\eta}([0, \tau] ; \mathbb{C}), g \in C^{1+\sigma}([0, \tau] ; \mathbb{C}),\left(z_{0} M+\right.$ $L) y_{0}+h(0) \in X_{A}^{\varphi}, \gamma, \varphi \in(5-3 \alpha-2 \beta, 1), \eta, \sigma \in((3-2 \alpha-\beta) / \alpha, 1), h \in C^{\mu}([0, \tau] ; X)$, where $\mu \in[\delta+(3-2 \alpha-\beta) / \alpha, 1), \delta \in I_{\alpha, \beta, \bar{\tau}}, \bar{\tau}=(\alpha+\beta+\gamma-2) / \alpha$,

$$
I_{\alpha, \beta, \bar{\tau}}= \begin{cases}\left(\frac{3-2 \alpha-\beta}{\alpha}, \bar{\tau}\right] & \text { if } \bar{\tau} \in\left(\frac{3-2 \alpha-\beta}{\alpha}, \frac{1}{2}\right) \\ \left(\frac{3-2 \alpha-\beta}{\alpha}, \frac{1}{2}\right) & \text { if } \bar{\tau} \in\left[\frac{1}{2}, 1\right) .\end{cases}
$$

Therefore, we can establish the result as follows.

Theorem 1. Assume $5 \alpha+2 \beta>6, z \in X_{A^{\prime}}^{\gamma} k \in C^{\eta}([0, \tau] ; \mathbb{C}), g \in C^{1+\sigma}([0, \tau] ; \mathbb{C}),\left(z_{0} M+L\right) y_{0}+h(0) \in$ $X_{A^{\prime}}^{\varphi} \gamma, \varphi \in(5-3 \alpha-2 \beta, 1), \eta, \sigma \in((3-2 \alpha-\beta) / \alpha, 1), h \in C^{\mu_{0}}([0, \tau] ; X)$, where $\mu_{0}-1 / 2 \in$ $[(3-2 \alpha-\beta) / \alpha, 1)$. Let $\bar{\tau}=\min (\sigma,(\alpha+\beta+\gamma-2) / \alpha)$. Then for any fixed $\delta \in I_{\alpha, \beta, \bar{\tau}}$ problem (14)-(16) admits a unique strict solution $(y, f)$ such that $v \in C^{\delta}([0, \tau] ; D(L)), M v \in C^{1+\delta}([0, \tau] ; X), f \in C^{\delta}([0, \tau] ; \mathbb{C})$.

\section{The General Case $\tilde{\alpha} \in(0,1)$}

In this section we handle problem (3)-(5) in the general case $\tilde{\alpha} \in(0,1)$. Without loss of generality, we consider the problem where $L$ is replaced by $z_{0} M+L$ (this can be justified by a simple change of variables). Now apply $\Phi$ to both sides of (3), taking into account (5), we obtain

$$
g^{(\tilde{\alpha})}(t)=\Phi\left[\left(z_{0} M+L\right) y(t)\right]+\int_{0}^{t} k(t-s) \Phi\left[L_{1} y(s)\right] d s+f(t) \Phi[z]+\Phi[h(t)],
$$

if $\Phi[z] \neq 0$, we get

$$
f(t)=\frac{1}{\Phi[z]}\left\{g^{(\tilde{\alpha})}(t)-\Phi\left[\left(z_{0} M+L\right) y(t)\right]-\int_{0}^{t} k(t-s) \Phi\left[L_{1} y(s)\right] d s-\Phi[h(t)]\right\},
$$

so that the inverse problem (3)-(5) is reduced to the following direct problem

$$
\begin{aligned}
& D_{t}^{\tilde{\alpha}}(M y(t))=\left(z_{0} M+L\right) y(t)-\frac{\Phi\left[\left(z_{0} M+L\right) y(t)\right]}{\Phi[z]} z+\int_{0}^{t} k(t-s) L_{1} y(s) d s-\int_{0}^{t} k(t-s) \frac{\Phi\left[L_{1} y(s)\right]}{\Phi[z]} z d s+ \\
& \\
& M y(0)=0 .
\end{aligned}
$$

Comparing this problem with (17), we conclude that our problem is solvable if we assume $g \in C^{\tilde{\alpha}(1+\theta)}([0, \tau] ; \mathbb{C}), h \in C^{\tilde{\alpha} \theta}([0, \tau] ; X), g^{(\tilde{\alpha})}(0)=h(0)=0, k \in C^{\tilde{\alpha} \theta}([0, \tau] ; \mathbb{C})$ in order to have $L y$, $D_{t}^{\tilde{\alpha}} M y \in C^{\tilde{\alpha} \omega}([0, \tau] ; X), \omega=\theta-2+\alpha+\beta$ and $f \in C^{\tilde{\alpha} \omega}([0, \tau] ; \mathbb{C})$, cfr. Section 3.

Theorem 2. Suppose that $g \in C^{\tilde{\alpha}(1+\theta)}([0, \tau] ; \mathbb{C}), h \in C^{\tilde{\alpha} \theta}([0, \tau] ; X), g^{(\tilde{\alpha})}(0)=h(0)=0, k \in$ $C^{\tilde{\alpha} \theta}([0, \tau] ; \mathbb{C})$. Then problem (11)-(13) admits a unique solution $(y, f) \in C^{\tilde{\alpha} \omega}([0, \tau] ; D(L)) \times C^{\tilde{\alpha} \omega}([0, \tau] ; \mathbb{C})$, where $\omega=\theta-2+\alpha+\beta$.

\section{Applications}

In this section we introduce two concrete cases of partial differential equations in which all our hypotheses run well and Theorem 2 can be applied. Of course, by using Favini and Yagi [27], many other concrete applications could be described. We begin with the following example. 
Example 1. Consider the inverse problem to find $(y, f)$ satisfying

$$
\begin{aligned}
& D_{t}^{\tilde{\alpha}}(m(x) y(t, x))=\Delta y(t, x)+\int_{0}^{t} k(t-s) \Delta y(s, x) d s+f(t) z(x)+h(t, x), t \in[0, \tau], x \in \Omega, \\
& m(x) y(t, x) \longrightarrow m(x) y_{0}(x) \text { in } L^{p}(\Omega) \text { as } t \rightarrow 0^{+}, 1<p<\infty, \\
& \Phi[m(x) y(t, x)]=\int_{\Omega} m(x) \sigma(x) y(t, x) d x=g(t), \sigma \in L^{p^{\prime}}(\Omega), 1 / p+1 / p^{\prime}=1,
\end{aligned}
$$

$\Omega$ being a bounded set in $\mathbb{R}^{n}$ with a smooth boundary, $k$ is continuous on $[0, \tau], m(x) \geq 0, m \in C(\bar{\Omega})$, $D(\Delta)=W^{2, p}(\Omega) \cap W^{1, p}(\Omega), \int_{\Omega} m(x) \sigma(x) y_{0}(x) d x=g(0), h$ sufficiently smooth. Of course the ambient space is $L^{p}(\Omega)$. The resolvent estimates hold with $\alpha=1, \beta=1 / p, p>1$. Similar situation is found in Favini and Yagi [27], pp. 79-80.

\section{Example 2. (Degenerate Parabolic Equation)}

Consider the inverse problem

$$
\begin{aligned}
& D_{t}^{\tilde{\alpha}} y=\Delta(a(x) y)+\int_{0}^{t} k(t-s) \Delta y(s, x) d s+f(t) z(x)+h(t, x), \quad t \in[0, \tau], \quad x \in \Omega, \\
& a(x) y(t, x)=0,(t, x) \in[0, \tau] \times \partial \Omega \\
& \left(g_{1-\tilde{\alpha}} * y\right)=0 \\
& \Phi[y(t, x)]=\int_{\Omega} \sigma(x) y(t, x) d x=g(t), a \in C(\bar{\Omega}), a(x) \geq 0,
\end{aligned}
$$

where $\Omega$ is a bounded domain in $\mathbb{R}^{n}, n \geq 1$, with a smooth boundary, the function $a(x) \geq 0$ on $\bar{\Omega}$ and $a(x)>0$ almost everywhere in $\Omega$, a being in $L^{\infty}(\Omega), \int_{\Omega} \sigma(x) y_{0}(x) d x=g(0), y(0, x)=y_{0}(x), x \in \Omega$, see Favini and Yagi [27], p. 81, Example 3.8. Using the change of variables $w=a(x) y$, with $m(x)=\frac{1}{a(x)}$, the above inverseproblem is reduced to

$$
\begin{aligned}
& D_{t}^{\tilde{\alpha}} m(x) w(t, x)=\Delta w(t, \cdot)+\int_{0}^{t} k(t-s) \Delta m(x) w(s, x) d s+f(t) z(x)+h(t, x), t \in[0, \tau], x \in \Omega, \\
& w(t, x)=0,(t, x) \in[0, \tau] \times \partial \Omega \\
& \left(g_{1-\tilde{\alpha}} * m w\right)=0 \\
& \Phi[m(x) w(t, x)]=\int_{\Omega} \sigma(x) m(x) w(t, x) d x=g(t), \sigma \text { fixed in } L^{2}(\Omega), L^{2}(\Omega) \text { istheambientspace. }
\end{aligned}
$$

One obtains a differential system to which the quoted results from Favini and Yagi [27] apply.

\section{Conclusions}

Some well known results for the case of non-fractional equations have been extended. Existence, uniqueness and regularity of solutions to the inverse problem related to degenerate fractional integro-differential equations have been studied. Some conditions on the underlying functions are imposed to guarantee the existence of a unique strict solution under less restrictive requirements than those presented in Reference [23,24], for example. This holds for Fedorov and Ivanova [7,13]. Applications from partial differential equations are given to illustrate our abstract results.

Author Contributions: Conceptualization, A.F.; methodology, M.A.H.; validation, M.A.H.; formal analysis, M.F., H.T.; investigation, M.A.H.; writing—original draft preparation, H.T.; writing—review and editing, M.F.; supervision, A.F.; project administration, M.F. All authors have read and agreed to the published version of the manuscript.

Funding: This research received no external funding. 
Conflicts of Interest: The authors declare no conflict of interest. The funders had no role in the design of the study; in the collection, analyses, or interpretation of data; in the writing of the manuscript, or in the decision to publish the results.

\section{References}

1. Al Horani, M.; Favini, A. An identification problem for first-order degenerate differential equations. J. Opt. Theory Appl. 2006, 130, 41-60. [CrossRef]

2. Al Horani, M.; Favini, A.; Tanabe, H. Direct and inverse fractional abstract Cauchy problems. Mathematics 2019, 7, 1016. [CrossRef]

3. Al Horani, M.; Fabrizio, M.; Favini, A.; Tanabe, H. Fractional Cauchy problems and applications. Discr. Contin. Dyn. Syst. Ser. S 2019. [CrossRef]

4. Al Horani, M.; Fabrizio, M.; Favini, A.; Tanabe, H. Fractional Cauchy problems for degenerate differential equations. Progr. Fract. Differ. Equ. Appl. 2019, 5, 1-11.

5. Al Horani, M.; Fabrizio, M.; Favini, A.; Tanabe, H. Identification problems for degenerate integro-differential equations. In Solvability, Regularity, and Optimal Control of Boundary Value Problems for PDEs; Springer INDAM Series; Springer: Berlin, Germany, 2017; Volume 22, pp. 55-75.

6. Favaron, A.; Favini, A.; Tanabe, H. Perturbation Methods for Inverse Problems on Degenerate Differential Equations. Available online: https://mathematicalanalysis.unibo.it/article/view/3422 (accessed on 25 March 2020).

7. Fedorov, V.E.; Ivanova, N.D. Identification problem for degenerate evolution equations of fractional order. Fract. Calculus Appl. Anal. 2017, 20, 706-721. [CrossRef]

8. Sviridyuk, G.A.; Fedorov, V.E. Linear Sobolev Type Equations and Degenerate Semigroups of Operators; VSP: Utrecht, The Netherlands; Boston, MA, USA, 2003.

9. Fedorov, V.E.; Urazaeva, A.V. An inverse problem for linear Sobolev type equations. J. Inverse Ill-Posed Probl. 2004, 12, 387-395. [CrossRef]

10. Gordievskikh, D.M.; Fedorov, V.E. Solutions of initial-boundary value problems for some degenerate systems of equations of fractional order in time. Bull. Irkutsk State Univ. Ser. Math. 2015, 12, 12-22.

11. Fedorov, V.E.; Gordievskikh, D.M. Resolving operators of degenerate evolution equations with fractional derivative with respect to time. Russ. Math. 2015, 59, 60-70. [CrossRef]

12. Fedorov, V.E.; Gordievskikh, D.M.; Plekhanova, M.V. Equations in Banach spaces with a degenerate operator under the sign of the fractional derivative. Differ. Equ. 2015, 51, 1360-1368. [CrossRef]

13. Fedorov, V.E.; Ivanova, N.D. Identification problem for a degenerate evolution equation with overdetermination on the solution semigroup kernel. Discr. Contin. Dyn. Syst. Ser. S 2016, 9, 687-696. [CrossRef]

14. Kiryakova, V. Generalized Fractional Calculus and Applications; Longman Scientific \& Technical: Harlow, UK, 1994.

15. Favini, A.; Lorenzi, A.; Tanabe, H. Degenerate integrodifferential equations of parabolic type with Robin boundary conditions: $L^{p}$-theory. J. Math. Anal. Appl. 2017, 447, 579-665. [CrossRef]

16. Favini, A.; Tanabe, H. Degenerate differential equations and inverse problems. In Proceedings of the Partial Differential Equations, Osaka, Japan, 21-24 August 2012; pp. 89-100.

17. Kostic, M. Abstract Volterra Integro-Differential Equations; Taylor and Francis Group, CRC Press: New York, NY, USA, 2015

18. Orlovsky, D.G. Parameter determination in a differential equation of fractional order with Riemann-Liouville fractional derivative in a Hilbert space. J. Sib. Fed. Univ. Math. Phys. 2015, 8, 55-63. [CrossRef]

19. Prilepko, A.I. The semigroup method for inverse, nonlocal, and nonclassical problems. Prediction-control and prediction-observation for evolution equations: I. Differ. Equ. 2005, 41, 1635-1646. [CrossRef]

20. Prilepko, A.I.; Orlovsky, D.G.; Vasin, I.A. Methods for Solving Inverse Problems in Mathematical Physics; Marcel Dekker: New York, NY, USA; Basel, Switzerland, 2000.

21. Glushak, A.V. On an inverse problem for an abstract differential equation of fractional order. Math. Notes 2015, 87, 654-662. [CrossRef]

22. Bajlekova, E.G. Fractional Evolution Equations in Banach Spaces; Eindhoven University of Technology: Eindhoven, The Netherlands, 2001. 
23. Balachandran, K.; Kiruthika, S. Existence of solutions of abstract fractional integrodifferential equations of Sobolev type. Comput. Math. Appl. 2012, 64, 3406-3413. [CrossRef]

24. Balachandran, K.; Kiruthika, S.; Trujillo, J.J. On fractional impulsive equations of Sobolev type with nonlocal condition in Banach spaces. Comput. Math. Appl. 2011, 62, 1157-1165. [CrossRef]

25. Favaron, A.; Favini, A. On the behaviour of singular semigroups in intermediate and interpolation spaces and its applications to maximal regularity for degenerate integro-differential evolution equations. Abstr. Appl. Anal. 2013, 2013, 37. [CrossRef]

26. Favini, A.; Lorenzi, A. Identification problems for singular integro-differential equations of parabolic type II. Nonlinear Anal. Theory Methods Appl. 2004, 56, 879-904. [CrossRef]

27. Favini, A.; Yagi, A. Degenerate Differential Equations in Banach Spaces; Marcel Dekker, Inc.: New York, NY, USA, 1999.

(C) 2020 by the authors. Licensee MDPI, Basel, Switzerland. This article is an open access article distributed under the terms and conditions of the Creative Commons Attribution (CC BY) license (http://creativecommons.org/licenses/by/4.0/). 